\title{
Hamsters remember spatial information derived from olfactory cues
}

\author{
W. THOMAS TOMLINSON and TIMOTHY D. JOHNSTON \\ University of North Carolina, Greensboro, North Carolina
}

\begin{abstract}
A dishabituation paradigm was used to study hamsters' memory for the spatial arrangement of olfactory cues. In Experiment 1, the animals dishabituated to a change in the positions of two different olfactory cues, but this experience did not reveal whether the response was based on egocentric (body-centered) or allocentric (body-independent) spatial information. In Experiment 2, the same dishabituation resulted when the experimental procedure required the animals to use spatial information that was independent of body position. Thus, hamsters remember the locations of olfactory cues with reference to a stable spatial framework. The dishabituation paradigm used here is a valuable tool for the study of animal spatial cognition, since it requires no explicit training and allows each animal to be tested in a relatively short experimental session.
\end{abstract}

In a number of open-field studies, it has been demonstrated that rodents are quite proficient at processing the spatial properties of their environment. However, in most of these studies, visual cues have been used to provide the rodent with spatial information. Our purpose in this study was to further elucidate the nature of spatial processing by testing adult hamsters (Mesocricetus auratus) for their ability to derive spatial information from olfactory cues.

Research on animal memory has involved a variety of different species in a number of experimental situations, including radial-arm mazes (Olton, 1979; Olton \& Samuelson, 1976) and place-learning mazes (Morris, 1984), open-field studies of primates (Menzel, 1973, 1978) and mice (Jamon \& Bovet, 1987), and ethological studies of insects (Hoffmann, 1985) and birds (Balda, 1980; Kamil \& Balda, 1985). All of this research indicates that memory for spatial information is highly developed among a wide range of animal species.

One widely held point of view about spatial memory (Menzel, 1978; Olton, 1979; Poucet, Chapuis, Durup, \& Thinus-Blanc, 1986) is that through experience the animal constructs a cognitive representation or schema of its spatial environment. This schema may organize the subsequent collection of new spatial information (Neisser, 1976), directing the animal's behavior toward novel spatial features of the environment. Memory for spatial information can thus be investigated by observing the dishabit-

This work was supported in part by a grant from the Research Council of the University of North Carolina at Greensboro to Timothy D. Johnston. The authors thank K. Smith, R. Shull, F. Russell, R. Fulk, and $\mathrm{L}$. Hyatt for their comments during the early stages of this project and during manuscript preparation. Reprint requests should be sent to either author. Mailing addresses: W. Thomas Tomlinson, Department of Psychology, University of Colorado, Boulder, CO 80309, or Timothy D. Johnston, Department of Psychology, University of North Carolina at Greensboro, Greensboro, NC 27412-5001. uation of exploratory behavior when spatial relationships among objects in the environment change in various ways. Dishabituation of exploration following a change in the spatial layout of objects occurs because the animal perceives a discrepancy between the current spatial configuration of objects in the environment and its memory for the previous layout (Neisser, 1976; Thinus-Blanc \& Ingle, 1985; Wilz \& Bolton, 1971). Thus, an animal's response, or lack of response, to a spatial change in its environment can be used to make inferences about stored spatial information. With a dishabituation paradigm, memory for the spatial location of single objects has been demonstrated in a number of rodents, including rats (Corman, Myer, \& Myer, 1967), hamsters (Poucet et al., 1986), and gerbils (Cheal, 1978; Thinus-Blanc \& Ingle, 1985). Poucet et al. (1986) used this paradigm to demonstrate that hamsters remember complex spatial relationships such as the topological relations among objects, the geometric patterns of objects, and the relations between objects and distal patterns.

Our aim in this study was to investigate the possibility that hamsters derive spatial information from olfactory cues in their environment. All of the studies cited above involved spatial mapping and visual cues: The animals were tested for their recognition of changes in the spatial relationships among visually conspicuous objects. However, most rodents possess well-developed olfactory systems, which suggests that olfactory mapping of the environment may be important in their spatial orientation. Jamon, Benhamou, and Sauve (1986) have suggested that rodents use the olfactory cues produced by vegetation in their home range to orient themselves toward their nests. Hamsters are known to use olfaction to guide early exploratory forays from the nest (Schoenfeld \& Leonard, 1985), and it seems likely that in adulthood they are able to remember complex spatial relationships among olfactory cues. 


\section{EXPERIMENT 1}

Experiment 1 was an attempt to determine whether hamsters can remember spatial information associated with olfactory cues.

\section{Method}

Subjects. The subjects were 12 adult hamsters (Mesocricetus auratus), 7 females and 5 males, born and reared in our laboratory colony. The hamsters were housed individually in clear polycarbonate cages $(22 \times 43 \times 21 \mathrm{~cm})$ containing laboratory animal bedding (Bed-O-Cob, Anderson Cob Division) and strips of newspaper for nest building, with food and water available ad lib. All animals were maintained on a reversed 14:10-h light:dark cycle, with lights on at $1500 \mathrm{~h}$.

Apparatus. Testing was carried out in a circular plastic arena $91.5 \mathrm{~cm}$ in diameter with sides $42 \mathrm{~cm}$ high to prevent possible drafts from disturbing the experimental odor cues. The arena was located in a dark testing room $(9.0 \times 6.9 \mathrm{~m})$ with the temperature maintained between $21.7^{\circ}$ and $24.4^{\circ} \mathrm{C}$. The arena was illuminated by a $60-\mathrm{W}$ bulb suspended $114 \mathrm{~cm}$ above the arena floor. The bulb was fitted with a domed shade designed to cast a shadow over the rest of the testing room. Patterns formed by these shadows may have provided visual cues for the hamsters. A white rectangle $(21.5 \times 37 \mathrm{~cm})$ painted with equally spaced, $4-\mathrm{cm}$-wide black vertical stripes was attached to the east wall of the arena, out of reach of the subjects, providing a conspicuous visual cue for orientation (Figure 1). The floor of the arena had an embossed pattern that may have provided additional visual or tactile cues for orientation.

Two objects (visual cues) and two odors (olfactory cues) were used in the study. The objects were two identical glass cheese shakers, $8.8 \mathrm{~cm}$ in height and $5.3 \mathrm{~cm}$ in diameter, both with perforated stainless steel caps. These objects provided a simple spatial array of olfactory cues with no visual differences. Each object was filled with sand for stability and had a square of gauze secured under its perforated cap for application of the odorant. Two floral potpourri oils (Carolina Fragrance Trading Company, Charlotte, NC), honeysuckle and gardenia, were used as odorants.

Procedure. Each subject underwent 1 day of testing and experienced only one of the two experimental conditions. On the day of testing, each subject was given a $15-\mathrm{min}$ familiarization trial in the empty test arena, followed by three 10-min experimental sessions (S1-S3). The subject was placed in the arena at the west wall at the beginning of the familiarization trial and all three experimental sessions. The hamster was returned to its home cage for $10 \mathrm{~min}$ between

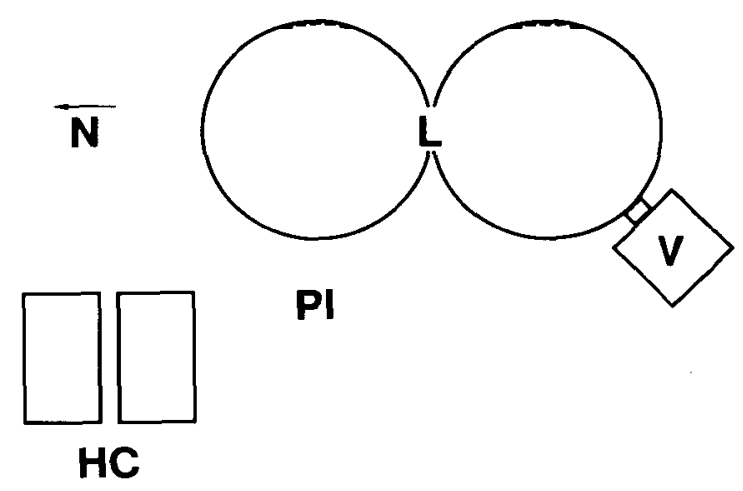

Figure 1. A schematic representation of the testing apparatus. $\mathrm{L}=60-\mathrm{W}$ light bulb; $\mathrm{V}=$ video camera; $\mathrm{PI}=$ location of observer during trials; HC = holding cage; $N=$ north.
Table 1

Means and Standard Errors for Contacts and Contact Times in Experiment 1

\begin{tabular}{lcccr}
\hline & \multicolumn{2}{c}{ Session 1 } & \multicolumn{2}{c}{ Session 2 } \\
\cline { 2 - 5 } Group & $M$ & $S E$ & $M$ & $S E$ \\
\hline & \multicolumn{5}{c}{ Contacts } \\
C & 23.50 & 3.55 & 10.00 & 1.18 \\
NC & 28.17 & 2.58 & 14.12 & 2.70 \\
& & Contact Times & & \\
C & 13.42 & 2.25 & 4.36 & 1.19 \\
NC & 13.67 & 2.46 & 4.92 & 1.46 \\
\hline
\end{tabular}

successive sessions. The subjects were randomly assigned to one of two groups: Group NC (no change) or Group C (change). For Group NC $(n=6)$, there was no change in the spatial layout of the arena between the second and third experimental sessions; for Group C $(n=6)$, the spatial layout was changed as described below.

Before the first session (S1), two cheese shakers, one scented with gardenia oil, the other with honeysuckle oil, were placed $25 \mathrm{~cm}$ apart along the north-south axis of the arena. The north-south placement of the odorants was counterbalanced across groups. Before the third session (S3), the location of the two cheese shakers and their associated olfactory cues was switched for Group C. Group NC encountered the same arrangement of olfactory cues as in S2. To control for the effects of moving the objects in Group C, and to be sure that the hamsters did not detect subtle changes in the object's location, the original objects were replaced before $\mathbf{S} 3$ for both groups. The replacement objects were identical in visual and olfactory properties to those in S2. A small fan was used to protect against excessive buildup of the odors between sessions. Before each session, the objects were wiped with a baking soda solution to remove any extraneous odors. After each animal had been tested, the arena was thoroughly cleaned and then wiped down with the baking soda solution to reduce the smell of the soap.

As in other studies (Poucet et al., 1986), a contact was recorded when the subject's snout touched the object. During each experimental session ( $S 1, S 2$, and $S 3$ ), the number of contacts that the animal made with each of the objects was counted, and the cumulative duration of these contacts was recorded with a silent hand-held stopwatch. All data were collected by an observer who sat $1 \mathrm{~m}$ from the arena's west wall. Interrater reliabilities for this procedure exceed 0.95 for contacts and 0.90 for contact times (Tomlinson, 1991).

Data analysis. Two dishabituation scores, one for number of contacts and one for cumulative contact times, were calculated for each subject by subtracting the number (or time) obtained in $\$ 2$ from the number (or time) obtained in S3.

\section{Results}

Every animal exhibited fewer contacts and less contact time in $\mathbf{S} 2$ than in $\mathbf{S 1}$, which suggests that they all habituated to the presence of the two objects in the arena (Table 1). Between S2 and S3, both the number of contacts and the contact time dishabituated in Group $\mathrm{C}$, but neither measure dishabituated in Group NC. The dishabituation scores for contact number and contact time (obtained by subtracting S2 scores from S3 scores) are shown in Figure 2. The animals in Group $\mathrm{C}$ had positive dishabituation scores on both measures, whereas Group NC's dishabituation scores were negative or zero. Analyses of variance (ANOVAs) performed on contact-number and contacttime dishabituation scores revealed statistically significant differences between groups in both cases [contact number, 

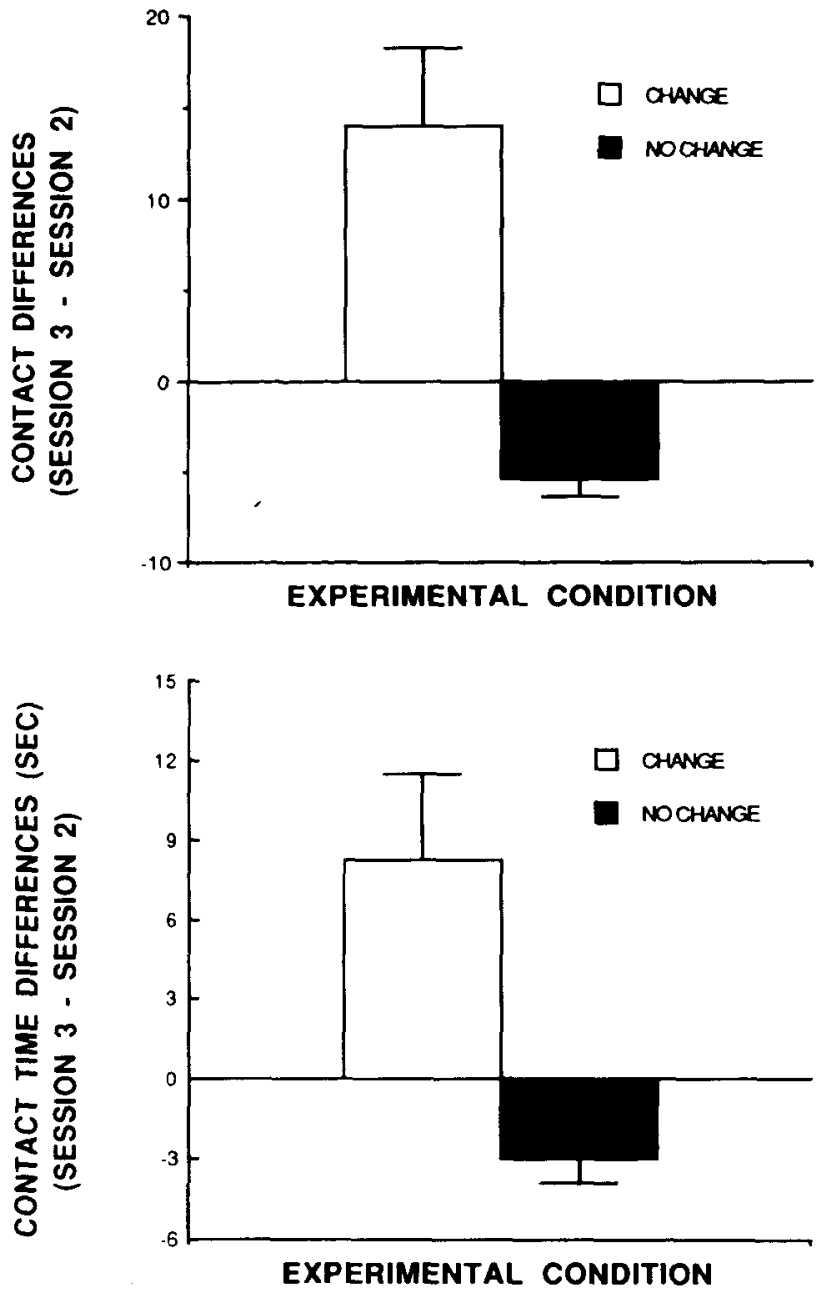

Figure 2. The mean dishabituation scores for Experiment 1. Top panel: mean number of contacts; bottom panel: mean contact time. (Dishabituation scores $=\mathbf{S 3}-\mathrm{S} 2$ contacts or contact times.) Note that the change group has positive scores, while the no-change group has negative scores (see text).

$F(1,10)=18.77, p=.0015$; contact time, $F(1,10)=$ $10.64, p=.0086$ ]. Further analyses revealed a statistically significant effect of experimental group on number of contacts in $S 3[F(1,10)=1.81, p=.0064]$, with Group $C$ having more contacts than Group NC (24.00 vs. 7.33). Group $C$ also had more time in contact with the objects during S3 than did Group NC (12.53 vs. 1.93) $[F(1,10)=16.67, p=.00022]$.

\section{Discussion}

The dishabituation of exploration in Group $\mathrm{C}$ during S3 demonstrates that hamsters increase their exploration of the environment when the position of olfactory cues changes and supports the hypothesis that hamsters represent spatial properties associated with those cues. The only feature of the arena that changed between S2 and S3 was the spatial location of the olfactory cues; the cues themselves and the objects with which they were associated remained the same. Thus we can infer (1) that the hamsters encoded the spatial locations of the olfactory cues during S1 and S2, and (2) that they recognized the change in spatial location of the cues that occurred between S2 and S3.

However, this experiment does not reveal very much about the properties of the hamsters' spatial memories of their olfactory environment, although it does demonstrate that such memories exist. Spatial information of at least two types might have been stored to produce the behavior seen during S3: information about the relation of the odors to the hamster's body position at the point of entry, or information about the position of the odors in the overall configuration of the space. Borrowing terms from the study of cognition in human infants (Pick \& Lockman, 1981), we call information about the spatial locations of objects in relation to the animal's own body coordinates egocentric spatial information. Information about the locations in relation to other spatial cues in the environment is called allocentric spatial information. The distinction between egocentric and allocentric spatial information corresponds to the earlier distinction between response learning and place learning made by Tolman (1948). Response learning requires only egocentric information, whereas place learning requires allocentric information.

To use allocentric spatial information, the animal must remember the configurations of objects in its environment, rather than simply remember the location of objects in relation to its own body position. This involves more sophisticated spatial information processing, since the animal must take into account a larger array of features of the environment. Thus, the hamster must remember the relationship between the two cues and at least one other cue in the arena or room, such as the striped card or patterns made by shadows outside of the arena. However, allocentric information is more useful to an animal than is egocentric information, since the appropriateness of a response based on allocentric information is not sensitive to changes in the position of the animal's own body. We would therefore expect animals to use allocentric rather than egocentric information whenever possible, but Experiment 1 provided no basis for distinguishing between the two. Either egocentric or allocentric information would have allowed the animals in Group $\mathrm{C}$ to detect the change in their environment. Our aim in Experiment 2 was to differentiate between these two types of spatial behavior by testing animals in a situation in which the use of egocentric or of allocentric information would lead to different predictions about the animals' behavior.

\section{EXPERIMENT 2}

In Experiment 1, the hamsters might have based their responses either on egocentric or on allocentric spatial information because they always entered the arena at the same location (the west wall). A hamster might have stored the information that honeysuckle (or gardenia) was on its 
right during the first two sessions and then detected the change when the odor was moved to the left in the third session. On the other hand, it might have stored a set of spatial relationships between the odors and some extramaze, intramaze, or combination of cues during $S 1$ and $S 2$, and then detected a change in those relationships in S3. In Experiment 2, we distinguished between these two types of stored information by introducing the animals into the arena at the start of $\mathrm{S} 3$ at a point $180^{\circ}$ from the point of entry for S1 and S2. Once again, the position of the two scented objects was changed for one group (Group C) between $\mathrm{S} 2$ and $\mathrm{S} 3$ but not for the other group (Group NC). If the animals were using allocentric information, exploratory behavior in Group C (but not in Group NC) should dishabituate in S3; if egocentric information was being used, the reverse should occur. Previous research on spatial orientation in hamsters (e.g., Poucet et al., 1986) has not distinguished between the use of these two kinds of spatial information.

\section{Method}

Subjects. Subjects were 16 hamsters (8 males and 8 females) housed under the same conditions as in Experiment 1. The subjects were randomly assigned to either Group NC (no change) or Group C (change) before testing.

Apparatus. The apparatus, objects, and odors were identical to those used in Experiment 1.

Procedure. The procedure was the same as in Experiment 1, except that at the start of $\$ 3$, animals were placed at the east wall of the arena (see Figure 1) rather than at the west wall, as they had been for S1 and S2. In Experiment 1, the animals started every session at the west wall. Data collection and analysis were the same as in Experiment 1.

\section{Results}

Fifteen of the 16 subjects exhibited fewer contacts and less contact time in S2 than in S1, showing that habituation to the two objects was successful (Table 2). When the animals were placed in the arena for S3, but now at the east rather than the west wall, both measures of exploratory behavior dishabituated in Group $C$ but not in Group NC. The dishabituation scores for contact number and contact time are shown in Figure 3. The animals in Group $\mathrm{C}$ had positive dishabituation scores on both measures; the scores of Group NC were negative or zero. ANOVAs revealed a significant difference between the groups in dishabituation of contact time $[F(1,14)=11.03$,

\section{Table 2}

Means and Standard Errors for Contacts and Contact Times in Experiment 2

\begin{tabular}{|c|c|c|c|c|}
\hline \multirow[b]{2}{*}{ Group } & \multicolumn{2}{|c|}{ Session 1} & \multicolumn{2}{|c|}{ Session 2} \\
\hline & $M$ & $\overline{S E}$ & $M$ & $\overline{S E}$ \\
\hline \multicolumn{5}{|c|}{ Contacts } \\
\hline $\mathrm{C}$ & 23.25 & 2.58 & 14.12 & 2.70 \\
\hline NC & 19.62 & 4.52 & 10.50 & 2.51 \\
\hline \multicolumn{5}{|c|}{ Contact Times } \\
\hline C & 9.92 & 1.59 & 4.53 & 1.46 \\
\hline $\mathrm{NC}$ & 9.75 & 3.33 & 5.22 & 1.86 \\
\hline
\end{tabular}
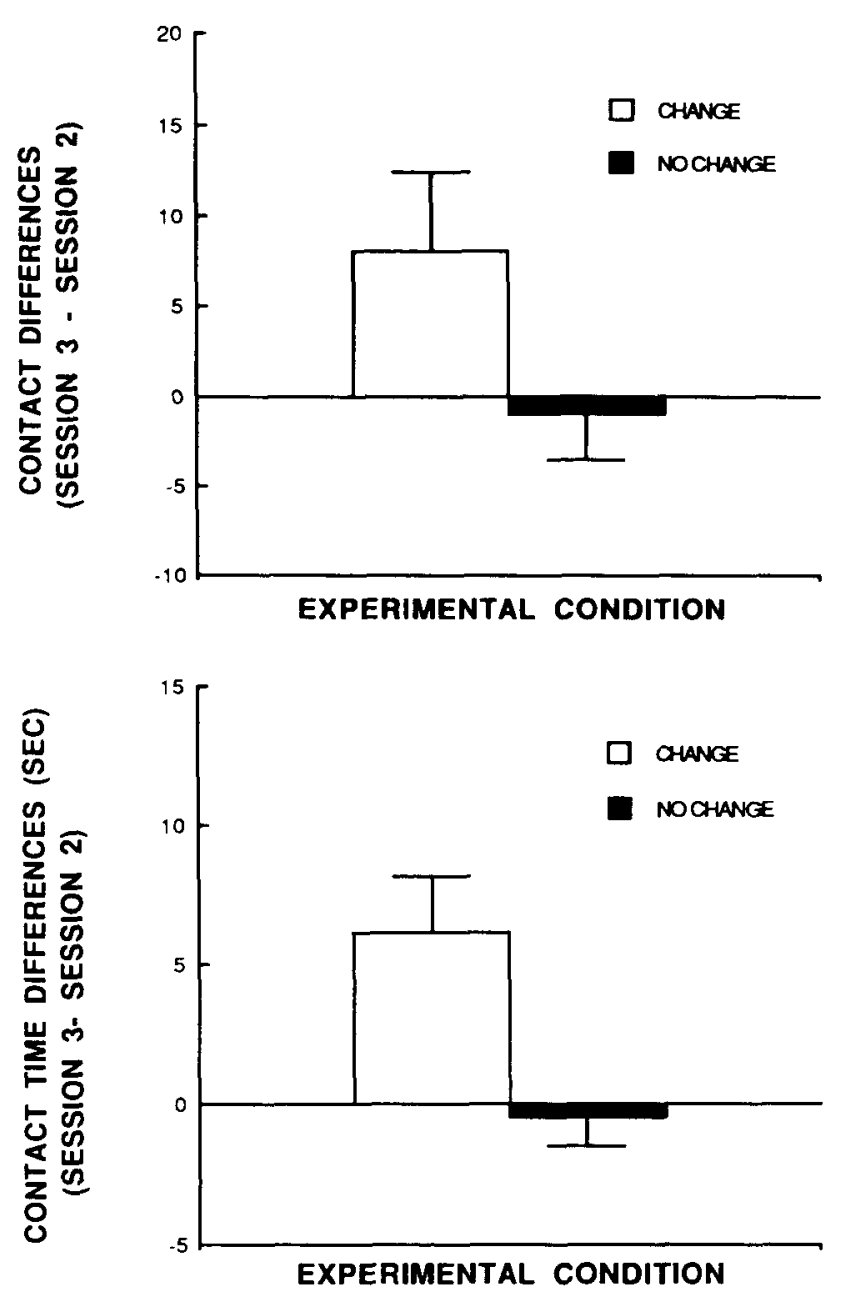

Figure 3. The mean dishabituation scores for Experiment 3. Top panel: mean number of contacts; bottom panel: mean contact time. Dishabituation scores $=\mathbf{S 3}-\mathbf{S 2}$ contacts or contact times. Note that the change group has positive scores, while the no-change group has negative scores (see text).

$p=.0050]$, but not contact number $[F(1,14)=3.79$, $p=.0720]$. However, an ANOVA did reveal a statistically significant effect of experimental group on number of contacts during $S 3[F(1,14)=4.90, p=.0439]$, with Group C having more contacts than Group NC did (22.12 vs. 9.38). Similarly, Group $C$ also had more time in contact with the objects than did Group NC [11.00 vs. 4.47 ; $F(1,14)=5.01, p=.0420]$.

\section{Discussion}

The finding that Group C, but not Group NC, dishabituated during $\mathrm{S} 3$ supports the hypothesis that the hamsters can use allocentric strategies and therefore must have stored configural information about the spatial location of the olfactory cues in the arena. If the hamsters had not stored configurational information, dishabituation would have been seen in Group NC rather than in Group C, since 
without configurational information the changed point of entry into the arena produces a change in the (egocentrically assessed) layout for the former group, but not for the latter.

\section{GENERAL DISCUSSION}

In this study, we used the dishabituation of exploratory behavior, a paradigm previously employed by Poucet et al. (1986) to study hamsters' spatial mapping of visual features, to investigate spatial mapping of olfactory cues in the environment. The first experiment demonstrated that hamsters remember the spatial relationships of olfactory features of their environment. The second experiment showed that hamsters use allocentric information in responding to changes in the spatial locations of olfactory cues; that is, they remember information derived from the odor cues and some other cue, probably the striped pattern.

The present study extends the findings of Poucet et al. (1986) by showing that hamsters remember spatial information associated with olfactory cues and by demonstrating that they store configurational information about the spatial location of those cues. Poucet et al. (1986) did not show that hamsters remember configurational information because their procedures could not rule out egocentric responding. Although their hamsters did demonstrate an ability to detect a change in the configuration of visual cues, the animals entered their experimental arena from the same direction on all trials (as in our Experiment 1). This is not a trivial point, since without a change in entry point between the last two sessions we cannot conclude that the hamsters remembered configurational information. The animals in our Experiment 1 and in Poucet et al.'s study would have responded in the same way had they been using egocentric (body-centered) information. Egocentric information is less adaptive than allocentric information, because its utility depends on the location of the animal at the time that the information is needed for subsequent responding.

The results obtained in this study confirm the view that when animals explore an environment they detect and encode spatial information from cues perceived during exploration. The spatial memories thus formed provide spatial information that may be useful for finding routes through the environment. Other investigators have drawn similar conclusions, using different approaches from that adapted here. Etienne and her colleagues (Etienne, Maurer, \& Saucy, 1988; Teroni, Portenier, \& Etienne, 1987) have investigated the cues used by hamsters to return to their nest after a foraging trip. They propose that proprioceptive information gained during the outward journey from the nest may guide the return in the absence of any sensory cues from the environment (Etienne et al., 1988). Chapuis, Durup, and Thinus-Blanc (1987) have demonstrated that hamsters can use stored spatial information to choose a novel route linking two previously unlinked subspaces, suggesting that hamsters in the wild may be able to determine novel routes between one location and the next on the basis of information in memory. Such an ability would be particularly adaptive for escaping predators, avoiding obstacles, or navigating in bad weather.

The animals in the present study were tested for incidental memory for spatial information. The animals were not trained or encouraged through any manipulation to learn the initial spatial layout of the odors, nor were they rewarded for recognizing the change in the spatial layout of the odor cues. Apparently, hamsters gather information about the spatial characteristics of an area during exploration and can use that information to control responding in subsequent situations. In other work (Tomlinson, 1991), this habituation paradigm has been used to study the development of the spatial abilities reported in this paper. In future investigations, we will examine how olfactory information is integrated with other sensory information during exploration.

\section{REFERENCES}

BALDA, R. P. (1980). Recovery of cached seeds by a captive Nucifraga caryocatactes. Zeitschrift fur Tierpsychologie, 52, 331-346.

Chapuis, N., Durup, M., \& Thinus-Blanc, C. (1987). The role of exploratory experience in a shortcut task by golden hamsters (Mesocricetus auratus). Animal Learning \& Behavior, 15, 174-178.

CheAL, M. L. (1978). Stimulus-elicited investigation in the Mongolian gerbil (Meriones unguiculatus). Journal of Biological Psychology, 20, 26-32.

Corman, C. D., Myer, P. M., Myer, D. R. (1967). Open-field activity and exploration in rats with septal and amygdaloid lesions. Brain Research, 5, 469-476.

Etienne, A. S., Maurer, R., Saucy, F. (1988). Limitations in the assessment of path dependent information. Behaviour, 106, 81-111.

HofFMANN, G. (1985). The influence of landmarks on the systematic search behaviour of the desert isopod Hemilepistus reaumuri. Behavioral Ecology \& Sociobiology, 17, 325-334.

Jamon, M., Benhamou, S., \& SAUVe, J. (1986). Initial orientation and navigation in homing rodents. In G. Beugnon \& I. E. C. Privat (Eds.), Orientation in space (pp. 45-55). Toulouse: Privat.

JAMON, M. , BOVET, P. (1987). Possible use of environmental gradients in orientation by homing wood mice, Apodemus sylvaticus. Behavioural Processes, 15, 93-107.

KAMIL, A. C., BAldA, R. (1985). Cache recovery and spatial memory in Clark's nutcrackers (Nucifraga columbiana). Journal of Experimental Psychology: Animal Behavior Processes, 11, 95-111.

MenzeL, E. W. (1973). Chimpanzee spatial memory organization. Science, 822, 943-945.

MenzeL, E. W. (1978). Cognitive mapping in chimpanzees. In S. H. Hulse, H. F. Fowler, \& W. K. Honig (Eds.), Cognitive aspects of animal behavior (pp. 375-422). Hillsdale, NJ: Erlbaum.

MorRIs, R. G. M. (1984). Development of a water-maze procedure for studying spatial learning in the rat. Joumal of Neuroscience Methods, 11, 47-60.

Neisser, U. (1976). Cognition and reality, San Francisco: W. H. Freeman.

Olton, D. S. (1979). Mazes, maps, and memory. American Psychologist, 34, 583-596.

Olton, D. S., \& SAmuelson, R. J. (1976). Remembrances of places passed: Spatial memory in rats. Journal of Experimental Psychology: Animal Behavior Processes, 2, 97-116. 
Pick, H. L., \& Lockman, J. (1981). From frames of reference to spatjal representations. In L. S. Liben, A. H. Patterson, \& N. Newcombe (Eds.), Spatial representation and the life span (pp. 39-61). New York: Academic Press.

Poucet, B., Chapuis, N., Durup, M., \& Thinus-Blanc, C. (1986). A study of exploratory behavior as an index of spatial knowledge in hamsters. Animal Learning \& Behavior, 14, 93-100.

SChOENFELD, T. A., \& LeONARD, C. M. (1985). Behavioral development in the Syrian golden hamster. In H. I. Siegel (Ed.), The hamster: Reproduction and behavior (pp. 289-321). New York: Plenum.

Teroni, E., Portenier, V., \& Etienne, A. S. (1987), Spatial orientation of the golden hamster in conditions of conflicting location-based and route-based information. Behavioral Ecology \& Sociobiology, 20, 389-397.
Thinus-Blanc, C., Ingle, D. (1985). Spatial behavior in gerbils (Meriones unguiculatus). Journal of Comparative Psychology, 99, 311-315.

Tolman, E. C. (1948). Cognitive maps in rats and men. Psychological Review, 55, 189-208.

TomLinson, W. T. (1991). Contribution of early exploratory experience to the development of olfactory spatial orientation in the golden hamster. Manuscript in preparation.

WiLz, K. J., \& Bolton, R. L. (1971). Exploratory behavior in response to the spatial rearrangement of familiar stimuli. Psychonomic Science, 24, 117-118.

(Manuscript received July 25, 1990;

revision accepted for publication January 3, 1991.) 